\title{
NEUTRAL HYDROGEN IN EXTRAGALACTIC SYSTEMS
}

\author{
LOUISE VOLDERS AND H. C. VAN DE HULST \\ Leiden Observatory, The Netherlands
}

In the past two years several extragalactic systems were observed for $21-\mathrm{cm}$

emission with the 25-meter telescope at Dwingeloo. The results on the Andromeda nebula (mainly points along the major axis) have already been published [1]. Observations of $M 33$ and M 101 are finished, a few other systems are still being observed.

The beamwidth of the Dwingeloo telescope at $21 \mathrm{~cm}$ is 0.57 degrees between half-power points. By alternately observing a measuring field and a comparison field for 15 -minute intervals, an accuracy of about $0.2^{\circ} \mathrm{K}$ in antenna temperature per measuring point was obtained. Each point gives at one frequency the intensity at one position relative to that in one or two comparison fields; it is the result of an hour's observation at a bandwidth of $150 \mathrm{kc} / \mathrm{s}$.

Table I gives the most relevant data obtained in Dwingeloo together with the Sydney data on the Magellanic Clouds. Differences from values published earlier [2] arise from (a) more recent observations, (b) revised distance estimates, and $(c)$ a revised temperature calibration for $\mathbf{M} 31$ consisting of an increase by 12 per cent.

$$
\text { M } 33
$$

The measured points along the major and the minor axis of $\mathrm{M} 33$, at distances of 0.25 degrees, and the comparison fields are shown in Fig. 1. The velocityintensity profiles drawn from these observations are shown in Fig. 1 with the following symbols:

Black dots: two comparison channels have been used, comparison field $V_{1}$, mean error $0.18^{\circ} \mathrm{K}$.

Black-white dots: one comparison channel has been used; comparison field $V_{1}$, mean error $0.24^{\circ} \mathrm{K}$.

Squares: measurements with two comparison fields. These were needed at velocities between -80 and $+20 \mathrm{~km} /$ second, since in that velocity range there may be a strong radiation from our own Galaxy. Linear interpolation between the two comparison fields seemed to give satisfactory results. Mostly $V_{1}$ and $V_{2}$ were used, but the points $x=+1, x=+2, x=+3$, and $x=+4$ were measured with nearby comparison fields, which were chosen symmetrically to the measuring field.

The further computation was made by the same method as used for M 31 . The nebula was supposed to be circularly symmetric. The hydrogen in the nebula was supposed to be concentrated near one plane and to be distributed in discrete rings at $a=1,2,3$, and a dot at $a=0$, where $a$ is the distance from the center in units of 0.25 degrees. The density distribution calculated from the area under the profiles at points on the major axis is given in Table II and Fig. 2. 


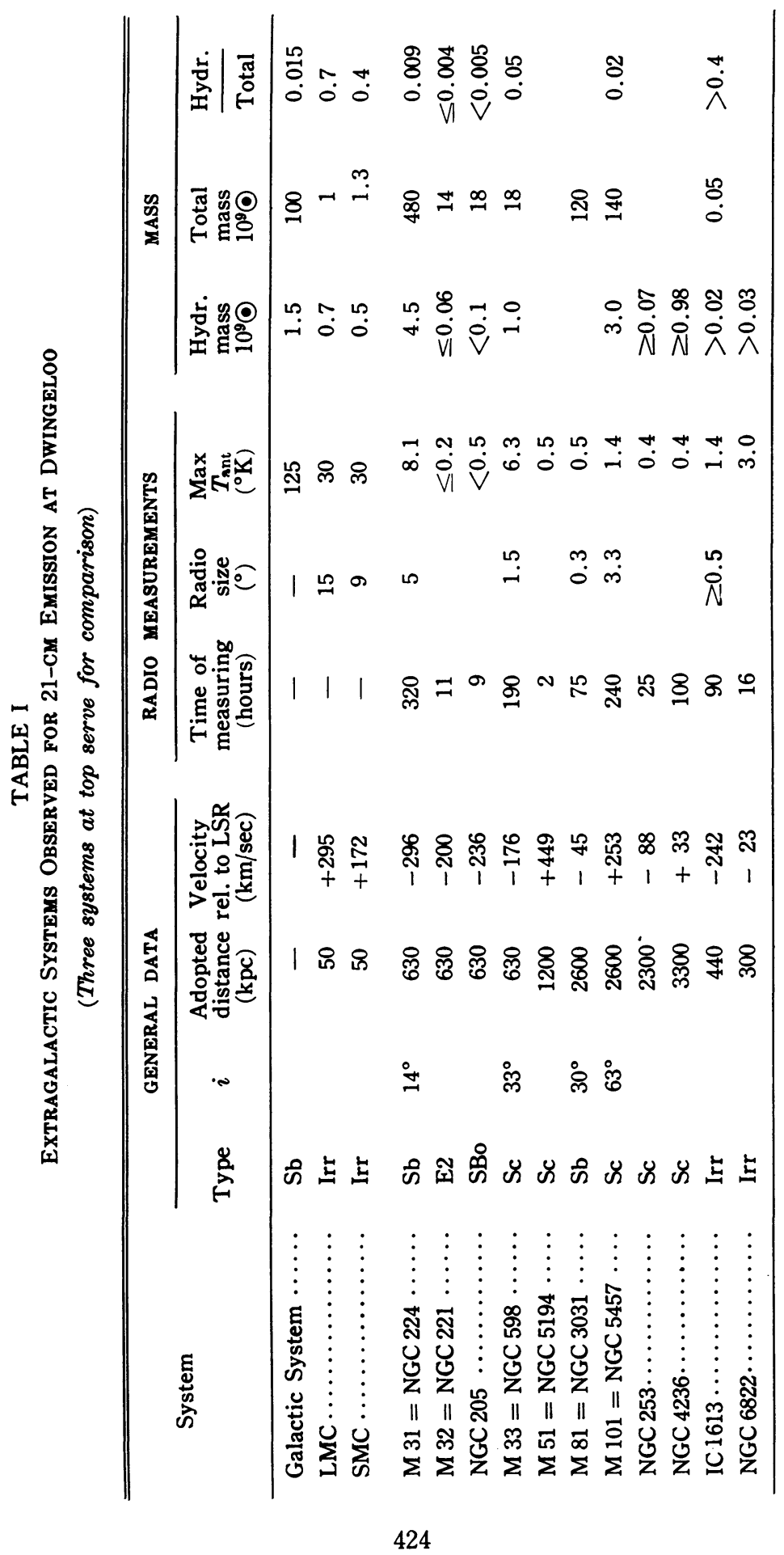



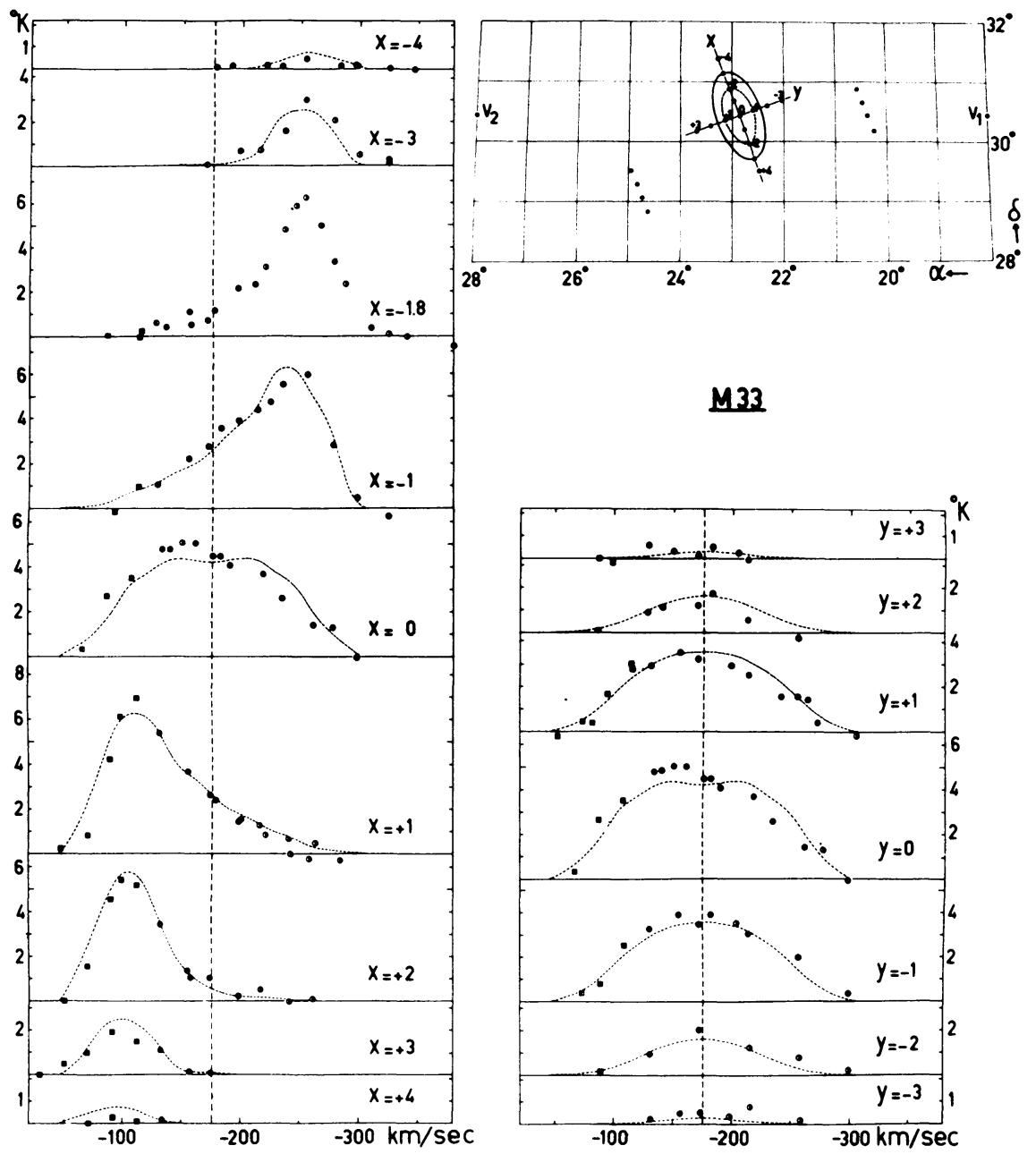

$\mathbf{M 3 3}$



FIG. 1. Observed profiles (dots) and computed profiles (dotted curves) for M 33. Insert shows position of measured points and comparison points.

TABLE II

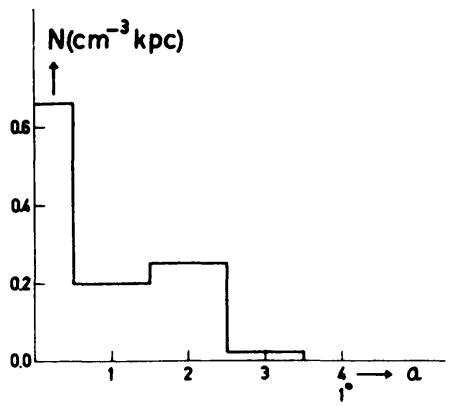

\section{33 DENSITY Distribution}

$\begin{array}{cc}a & \bar{N}(a) \\ (0.25) & \left(\mathrm{cm}^{-3} \mathrm{kpc}\right) \\ 0 & 0.66 \\ 1 & 0.20 \\ 2 & 0.25 \\ 3 & 0.02\end{array}$

FIG. 2. Density distribution of M 33. 


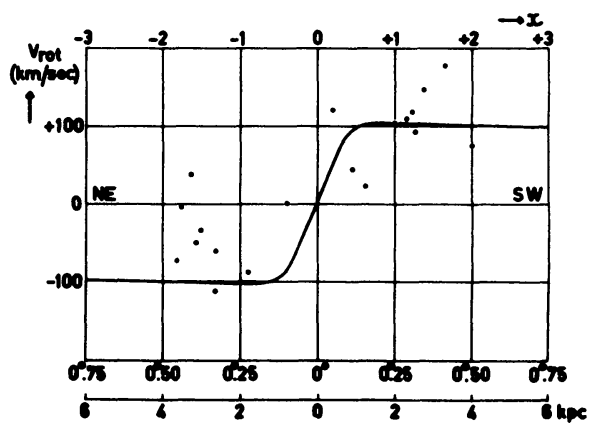

FIG. 3. Rotation law of M 33 ; dots are optical data. (The scale in kpc corresponds to the originally adopted distance of $460 \mathrm{kpc}$ and should be multiplied by 1.37 to agree with the distance adopted in Table I.)
TABLE III

M 33 ROTATION LAW

$\begin{array}{ccc}\begin{array}{c}a \\ (0.25)\end{array} & \begin{array}{c}V(a) \\ (\mathrm{km} / \mathrm{sec})\end{array} & \begin{array}{c}V_{\text {rot }} \\ (\mathrm{km} / \mathrm{sec})\end{array} \\ 4 & 50 & 60 \\ 1 & 86 & 102 \\ 2 & 84 & 100 \\ 3 & 82 & 98\end{array}$

The velocity of the center of gravity was found to be $-176 \pm 2 \mathrm{~km} / \mathrm{second}$. This is in good accordance with the optical data which are $-190 \mathrm{~km} / \mathrm{second}$ [8] and $-168 \mathrm{~km} / \mathrm{second}$ [4] with respect to the local standard of rest. The rotation law that was found to give a good agreement of theoretical

and observed profiles is presented in Table III and Fig. 3. The velocities $V(a)$ are the rotational velocities in the line of sight, and have to be multiplied by $\sec i=1.19$ to obtain the rotation in the plane of the nebula $V_{\text {rot. }}$ With this density distribution and rotation curve, theoretical line profiles were calculated for the major and minor axes (dotted curves).

The hydrogen mass, calculated with this model and at a distance of 630 kiloparsecs is $0.92 \times 10^{9}$ solar masses. The total mass estimated from the rotation curve is $18 \times 10^{9}$ solar masses.

The results obtained on the same system at Harvard [5] with different equipment and a different method of observation suggest more detail in the velocity profiles and a larger size of the radio nebula. In order to test the difference in method we made sets of drift-curves with the reduced time constant of 30 seconds at declinations and velocities coinciding with Figs. 2 and 8 of Mrs. Dieter's thesis. These tests showed that the drift-curve method can indeed be quite useful. By taking 1-minute averages of four drift-curves a mean error of $0.30^{\circ} \mathrm{K}$ could be attained in good agreement with the theoretical formula. Fig. 4 shows a sample of such a set of drift-curves and their average curve. The squares at the points where it crosses the major and minor axes indicate the values found by interpolation

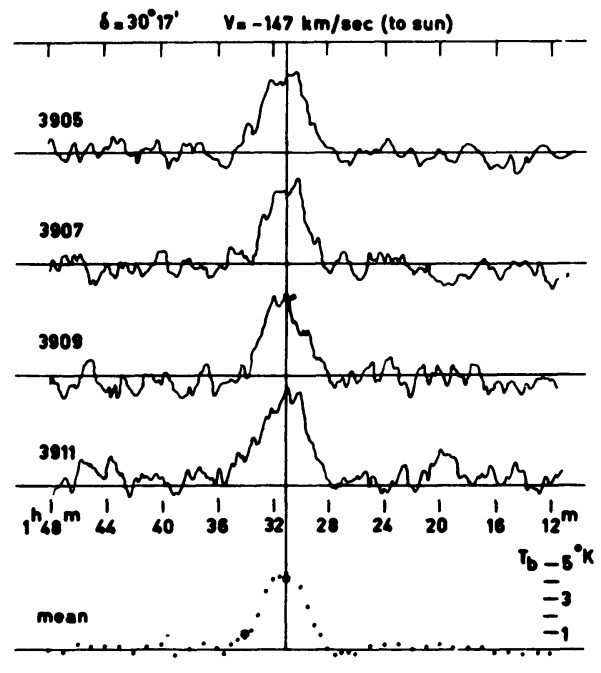

FIg. 4. Four sample drift curves on $M 33$ taken at the same declination and velocity. 
from Fig. 1 and demonstrate the internal consistency of these measurements. The receiver used by Mrs. Dieter apparently did not permit observations of comparable accuracy ([5], Fig. 8).

\section{101}

At first sight, this nebula seems to be perpendicular to the line of sight. By experimenting with oblique projection to make it look round we found that $i=63$ degrees and that the position angle of the major axis is 30 degrees. Observations of the $21-\mathrm{cm}$ line at six points around the center (points $A, B,+1, C, D,-1$ in Fig. 5) gave an independent determination of the major axis. An asymmetry measure was defined for these points as $f=$ average

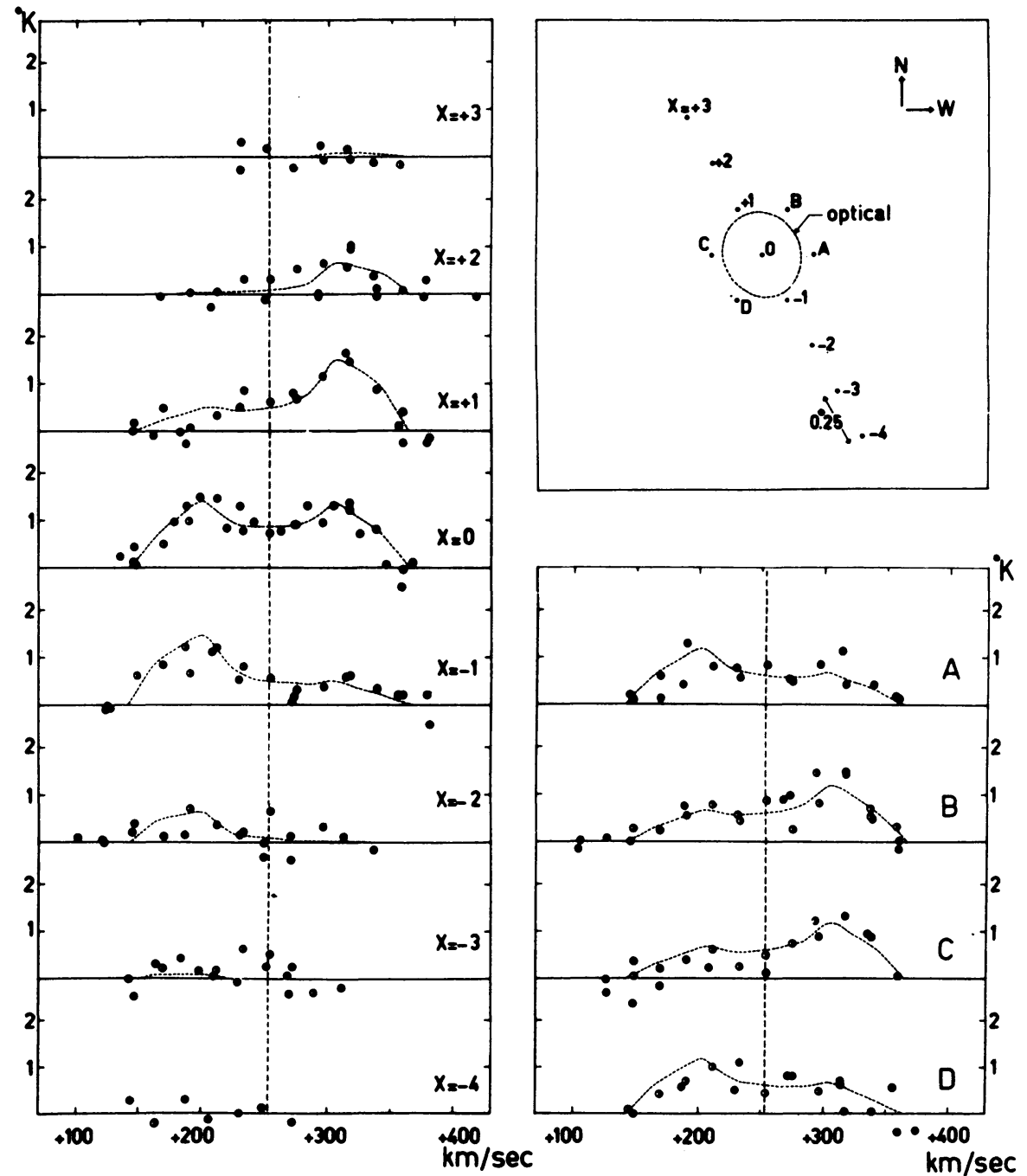

FIG. 5. Observed profiles (dots) and computed profiles (dotted curves) for $\mathrm{M} 101$. Insert shows position of measured points. 
$T$ in velocity interval 153 to 233 minus average $T$ in interval 273 to 353 . The values for the six points are $100 f=3,45,92,78,-28,-53$; by plotting them against position angle and fitting a sine curve we found position angle of major axis $=30 \pm 9$ degrees (m. e.).

Further observations along the major axis were made and reduced in the same manner as for M 33 . Fig. 5 gives the detailed results.

Table IV gives the density distribution.

TABLE IV

$\begin{array}{cc}\text { M } 101 & \text { DENSITY DistributioN } \\ a & \bar{N}(a) \\ (0.25) & \left(\mathrm{cm}^{-3} \mathrm{kpc}\right) \\ 0 & 0.33 \\ 0.42 & 0.20 \\ 0.84 & 0.22 \\ 1.26 & 0.02 \\ 1.68 & 0.02\end{array}$
The hydrogen mass is $3.0 \times 10^{9}$ solar masses. The velocity of the center is $253 \pm 3 \mathrm{~km} / \mathrm{second}$. The rotation in the line of sight is about $80 \mathrm{~km} / \mathrm{second}$, that is $178 \mathrm{~km} / \mathrm{second}$ in the plane of the nebula. Theoretical profiles are shown as dotted curves.

Since the rotation is very uncertain in the outer rings $(a=1.26$ and $a=1.68)$ and since also the inclination is not known with great accuracy, it is difficult to estimate the total mass. However it is certain that the mass of M 101 is much larger than the mass of M 33 and, therefore, that M 33 has relatively more hydrogen than M 101.

IC 1613

The center profile of this irregular system gave a maximum antenna temperature of $0.5^{\circ} \mathrm{K}$. However, a higher value of $1.5^{\circ} \mathrm{K}$ was found at points 0.5 degrees east and northeast of the center, where photographs show nothing. As the limits of the radio nebula have not yet been discovered only a minimum estimate of the hydrogen mass is given.

NGC 6822

Another promising object, this one is difficult to observe because of its small velocity and small galactic latitude. So far only the center has been observed.

\section{81}

The hydrogen radiation from this system is barely detectable. One point at a distance of $\frac{1}{8}$ degree from the center gives a positive result with $T_{\max }=$ $0.5^{\circ} \mathrm{K}$. Three other observed points give zero result.

\section{REFERENCES}

[1] van de Hulst, H. C., Raimond, E., and van Woerden, H. B.A.N. 14, 1, 1957.

[2] van de Hulst, H. C. Publication of Solvay Congress, Brussels 1958. (In press.)

[3] Humason, M. L., Mayall, N. U., and Sandage, A. R. A.J. 61, 97, 1956.

[4] Mayall, N. U., and Aller, L. H. Ap. J. 95, 5, 1942.

[5] Dieter, N. H. Doctoral thesis, Harvard University, March 1958. 


\section{Discussion}

van de Hulst: There sometimes has been a feeling that the radio-astronomical field of study before us is so vast that a strict division of labor between observatories would be advisable. It would be dangerous to adopt this attitude for any subject of which the measurements are really difficult. It is far more desirable that two or more observatories pursue the same course of investigations in order to provide cross-checks. Therefore, we are quite happy that Harvard Observatory has gone ahead with the measurements on the $21-\mathrm{cm}$ line from other galaxies. They already provide valuable checks on the order of magnitude of the radiation received.

Brown: Several of these external galaxies lie in regions of the sky where the isophotes of continuum radiation at $1.9 \mathrm{~m}$ show complicated patterns due possibly to galactic or extragalactic radiation. It would be valuable to check these measurements of hydrogen radiation from galaxies, in a few selected cases, by using drift-curves over a large region around those galaxies or by using a large number of comparison fields. I should like somebody to check whether or not the complicated patterns of continuum radiation that we see are associated with the galaxies.

van de Hulst: A careful check of this point is indeed necessary. We have used two comparison fields at any frequency at which we suspected that galactic radiation might bother us. Also we have intercompared the comparison fields used for M 31 and M 33, and the difference was negligible for a range of frequencies.

Margaret Burbidge: The proportion $f$ of the total masses of galaxies in the form of hydrogen is correlated with the total mass of the galaxies as well as with nebular type. This can perhaps be regarded as a correlation between nebular type and mass. At any rate, the greater the total mass, the greater is the proportion of it that has been condensed into stars. I should like to ask whether Dr. Schmidt has any comments on this?

Schmidt: It seems that the rate of star formation-and therefore the rate of depletion of gas-depends on a power of the gas density which is higher than unity. Consequently, the percentage of gas decreases faster in the denser, massive systems. I would expect then that in these systems $f$ will be lower.

Zirin: Is it possible to look for expansion in extragalactic systems?

van de Hulst: The angular resolution of the present telescopes does not make it very promising to look for expansion. The part of the galactic system out to 3 kiloparsecs, if put at the distance of M 31, would just about fill the beam. And on the minor axis, where one should look for expansion the situation is even four times less favorable.

Bidelman: Does Dr. van de Hulst have any estimate of the amount of ionized or molecular hydrogen in the galaxies in which he has determined the amount of neutral hydrogen?

van de Hulst: The ionized hydrogen will be discussed in Westerhout's 
paper. The molecular hydrogen, unfortunately, is unobservable, so except for theoretical arguments, the question is entirely open.

Ort: Would Dr. Mayall care to tell about the optical evidence?

Mayall: I have no new information on the distribution of ionized hydrogen in M 33 from spectrographic observations. But I think that many more observations of radial velocities of $\mathrm{H}$ II regions, with greater dispersion, would be a useful contribution to investigations of the distribution of mass. In noting the comparison of the modern radio observations of rotation with the 20-year-old optical data, I am not troubled too much by the lack of agreement; rather, I am surprised that the agreement is so good, considering the low precision of the optical radial velocities.

Gold: The amount of hydrogen in ionized form that may be overlooked cannot be very large, since its density must be low and the volume of the regions limited. It is different with molecular hydrogen, the density of which in cold, dusty regions may be very high. In these regions we have as yet no information to place a limit other than that obtained from the gravitational effects of such material; and such a limit is still very wide.

Hoyle: Isn't it also true that the mass of hydrogen will be underestimated if much of the hydrogen is concentrated in small clouds that are saturated in the $21-\mathrm{cm}$ line?

van de Hulst: I fully agree that this is an uncertainty. Denser and cooler regions can, in principle, be recognized as regions of low intensity. Certain attempts have already been made in that direction. Very small and dense clouds would pass, as you say, unnoticed. But this holds for the galactic system as well as for the other systems of the list, so the comparison is fair.

Bok: The relatively low density of neutral and ionized atomic hydrogen in the region near the sun is rather surprising. Recent work has suggested that the original estimate of 0.09 to 0.10 solar masses/parsec $^{3}$ for the OortKapteyn limit is on the low side and that 0.14 is a more reasonable figure. Of this the stars account for at most 0.07 , the hydrogen 0.02 , with the dust responsible for an insignificant fraction of the total. What is the remainder? We should not discount the possibility that it may be molecular hydrogen. The recent ultraviolet rocket research at the U.S. Naval Research Laboratory shows strong background radiations occurring in regions like the Orion complex out to distances where ionized hydrogen can hardly be present. The infrared opens up other possibilities. One can truthfully say that our inability to observe molecular hydrogen in interstellar space represents one of our greatest handicaps in the study of the physics and evolution of the interstellar gas-and every effort should be made to remedy this inability.

Oort: The present evidence indicates a somewhat higher mass density near the sun, which probably cannot be accounted for by the known stars. But it does not mean that this excess must consist of hydrogen. For instance, we know that in some elliptical galaxies considerable mass must exist in addition to the mass provided by the luminous stars, and in this case it is unlikely that it would consist of interstellar gas. 